\title{
AN UNSUPERVISED METHOD FOR REAL TIME VIDEO SHOT SEGMENTATION
}

\author{
Hrishikesh Bhaumik ${ }^{1}$, Siddhartha Bhattacharyya ${ }^{2}$ and Susanta \\ Chakraborty $^{3}$ \\ ${ }^{1,2}$ Department of Information Technology, RCC \\ Institute of Information Technology, Kolkata, India \\ hbhaumikegmail.com, dr.siddhartha.bhattacharyya@gmail.com \\ ${ }^{3}$ Department of Computer Science and Technology, \\ Bengal Engineering and Science University, Shibpur, Howrah, India \\ susanta.chak @gmail. com
}

\begin{abstract}
Segmentation of a video into its constituent shots is a fundamental task for indexing and analysis in content based video retrieval systems. In this paper, a novel approach is presented for accurately detecting the shot boundaries in real time video streams, without any a priori knowledge about the content or type of the video. The edges of objects in a video frame are detected using a spatio-temporal fuzzy hostility index. These edges are treated as features of the frame. The correlation between the features is computed for successive incoming frames of the video. The mean and standard deviation of the correlation values obtained are updated as new video frames are streamed in. This is done to dynamically set the threshold value using the three-sigma rule for detecting the shot boundary (abrupt transition). A look back mechanism forms an important part of the proposed algorithm to detect any missed hard cuts, especially during the start of the video. The proposed method is shown to be applicable for online video analysis and summarization systems. In an experimental evaluation on a heterogeneous test set, consisting of videos from sports, movie songs and music albums, the proposed method achieves $99.24 \%$ recall and $99.35 \%$ precision on the average.
\end{abstract}

\section{KEYWORDS}

Real time video segmentation, spatio-temporal fuzzy hostility index, image correlation, threesigma rule

\section{INTRODUCTION}

There has been a spectacular increase in the amount of multimedia content transmitted and shared over the internet. The number of users for video-on-demand and Internet Protocol Television (IPTV) services are growing at an exponential rate. According to 2012 statistics, there were more than one million IP-TV streams per month on the Zatoo platform. Textual annotation i.e. associating a set of keywords for indexing multimedia content was performed to facilitate searching of relevant information in existing video repositories (e.g. YouTube, DailyMotion etc.). However, manual annotation of the millions of videos available in such repositories is a cumbersome task. Content based video analysis techniques [1, 3, 4, 5, 6, 7, 8, 9] do away with manual annotation of the video data and results in saving time and human effort, with increase in accuracy. Video segmentation is the preliminary step for analysis of digital video. These segmentation algorithms can be classified according to the features used, such as pixel-wise

Dhinaharan Nagamalai et al. (Eds) : ACITY, WiMoN, CSIA, AIAA, DPPR, NECO, InWeS - 2014 pp. 307-318, 2014. (C) CS \& IT-CSCP 2014

DOI : $10.5121 /$ csit.2014.4531 
difference [10], histograms [11], standard deviation of pixel intensities [12], edge change ratio [13] etc. A more daunting task arises for online analysis and indexing of live streaming videos such as telecast of matches or live performances. Generation of highlights or summarization of such events is a challenging task as it involves development of algorithms which have good performance and are adapted to work in real time. Although video edits can be classified into hard cuts, fades, wipes etc. [9], the live streams mainly contain hard cuts, which is the main focus of the proposed approach. Several methods for video segmentation $[8,10,11,13,14]$ exist in the literature, which have been applied for non-real time videos. However, shot boundary detection for streaming videos is hard to find in the literature. The difficulties and problems related to setting thresholds and the necessity of automatic threshold have been discussed in [1]. Hence, the motivation behind this work was to develop a technique for video segmentation, which is able to detect with high accuracy, the hard cuts present in live streaming videos. Video segmentation is performed on the fly, as new video frames are streamed in. Also, in this work, the problem of setting an automatic threshold has been addressed. The threshold is set dynamically without any $a$ priori knowledge about the type, content or length of the video. The algorithm incorporates a look back mechanism to detect any missed hard cuts particularly during the start of the video when the statistical measures used to set the threshold are unstable. The novelty of the proposed work lies in its applicability in video summarization tasks of real time events, such as producing highlights of sports videos.

The reminder of the paper is organized as follows. In section 2, the basic concepts and definitions are presented. The proposed method for real time video segmentation is described in section 3 . The experimental results and analysis are presented in section 4. The comparison with other existing approaches is also given in the same section. Finally, the concluding remarks are mentioned in section 5 .

\section{BASIC CONCEPTS AND DEFINITIONS}

\subsection{Application of fuzzy set theory to image processing}

A colour image may be considered as a 3D matrix of values where each pixel colour depends on the combination of RGB intensities. Conversion of the colour image to a gray scale image involves mapping of the values in the $3 \mathrm{D}$ matrix to a $2 \mathrm{D}$ matrix, where each pixel value is in the range $[0,255]$. This $2 \mathrm{D}$ matrix of values may be scaled to the range $[0,1]$ by dividing each element of the matrix by 255 . The scaled value represents the membership value of each pixel to the fuzzy sets labelled as WHITE and BLACK. The number of elements in each fuzzy set is equal to the number of elements in the said 2D matrix. If a value 0 represents a completely black pixel and 1 a completely white one, then value of each element depicts the degree of membership $\mu_{W}\left(p_{i}\right)$ of the $i^{\text {th }}$ pixel $p_{i}$ to the fuzzy set WHITE. The degree of membership $\mu_{B}\left(p_{i}\right)$ of the pixel $p_{i}$ to the set BLACK can be represented as $\mu_{B}\left(p_{i}\right)=1-\mu_{W}\left(p_{i}\right)$. Incorporating the fuzzy set theory, Bhattacharyya et al. [2] have proposed the fuzzy hostility index for detecting the point of interest in an image, which is useful for high speed target tracking. As an extension of this concept, a new index is proposed for obtaining the edge map of a video frame as explained in the next sub-section.

\subsection{Edge Map using Spatio-Temporal Fuzzy Hostility Index (STFHI)}

Fuzzy hostility index [2] indicates the amount of variation in the pixel neighbourhood with respect to itself. The pixel hostility has high value if the surrounding pixels have greater 
difference of values as compared to the candidate pixel i.e. heterogeneity in its neighbourhood is more. In an $n$-order neighbourhood the hostility index $(\zeta)$ of a pixel is defined as:-

$\zeta=\frac{3}{2^{n+1}} \sum_{i=1}^{2^{n+1}} \frac{\left|\mu_{p}-\mu_{q i}\right|}{\left|\mu_{p}+1\right|+\left|\mu_{q i}+1\right|}$

where $\mu_{p}$ is the membership value of the candidate pixel and $\mu_{q i} ; \mathrm{i}=1,2,3, \ldots, 2^{n+1}$ are the membership values of its fuzzy neighbours in a second-order neighbourhood fuzzy subset. The value of the fuzzy hostility index $\zeta$ lies in $[0,1]$, with $\zeta=1$ signifying maximum heterogeneity and $\zeta=0$ indicating total homogeneity in the neighbourhood. The concept of fuzzy hostility index can be effectively extended to accommodate temporal changes in the time sequenced frames of a video.

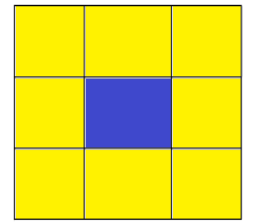

(a)

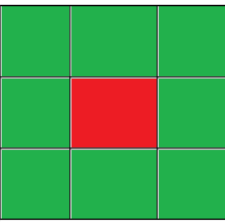

(b)

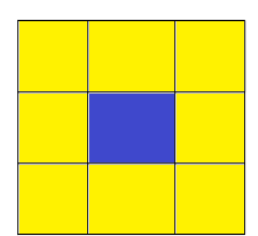

(c)

Figure 1. (a) Pre-frame $\left(f_{i-1}\right)$ (b) Present frame $\left(f_{i}\right)$ (c) Post-frame $\left(f_{i+1}\right)$

The STFHI $(\lambda)$ of a pixel in the $i^{\text {th }}$ image frame $f_{i}$ of a video is a function $\boldsymbol{\omega}$ of the fuzzy hostility index of the candidate pixel in $f_{i}$ (marked red in figure 1(b)) and the corresponding pixels in the previous $f_{i-1}$ and post $f_{i+1}$ frames (marked blue in figures 1(a) and 1(c)), can be expressed as follows:- $\lambda_{f_{i}}=\omega\left(\zeta_{f_{i-1}}, \zeta_{f_{i}}, \zeta_{f_{i+1}}\right)$

In other words, $\lambda$ of a pixel is a function of the second order neighbourhood of its corresponding pixels (marked yellow in figures 1 (a) and 1(c)) and itself (marked green in figure 1(b)). $\lambda_{f_{i}}$ is computed as the average of $\zeta_{f_{i-1}}, \zeta_{f_{i}}$ and $\zeta_{f_{i+1}}$ except for the first and last frames of a video where $\zeta_{f_{i-1}}$ and $\zeta_{f_{i+1}}$ are not present respectively. The $2 \mathrm{D}$ matrix thus formed by computing the $\lambda$ of each pixel will represent the edge map of an image with profound edges of all objects present in the original image as depicted in figure 3(b).

\subsection{Pixel Intensification Function}

Pixel intensity scaling is performed to make the edges more prominent compared to other portions of the image as shown in figure 3(c). The intensity scaling function $(\phi)$ used in this work is shown in figure 2 and mathematically represented as:-

$$
\begin{aligned}
\phi & =\left(\lambda_{i j}\right)^{2}, \text { if } \lambda_{i j}<0.5 \\
& =\left(\lambda_{i j}\right)^{1 / 2}, \text { if } \lambda_{i j} \geq 0.5
\end{aligned}
$$

where $\lambda_{i j}$ is the STFHI of the pixel at $i^{\text {th }}$ row and $j^{\text {th }}$ column. 


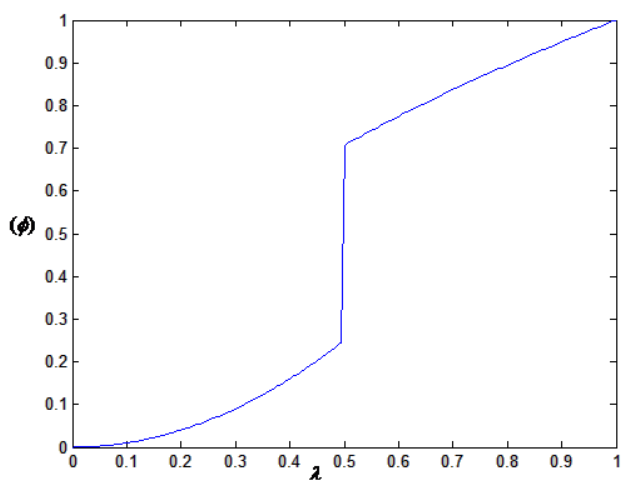

Figure 2. Pixel Intensity Function

\subsection{Edge Dilation}

Edge dilation is a technique which is used to enlarge the boundaries of the objects in a grayscale image as depicted in figure 3(d). This may be used to compensate for camera and object movement. A $3 \times 3$ square structuring element is used to dilate the edges of the grayscale image so generated from the fuzzy hostility map. The value of correlation between the similar images is increased as a result of edge dilation.

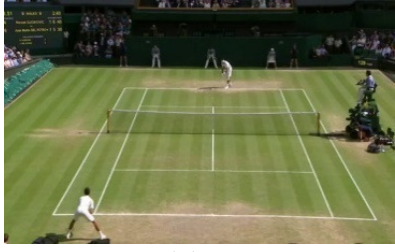

(a)

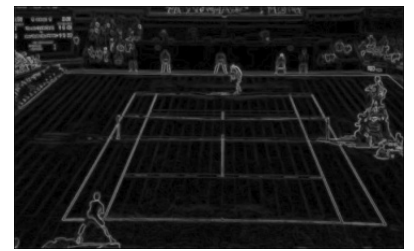

(c)

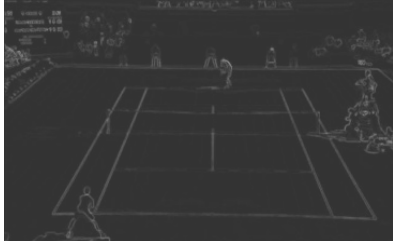

(b)

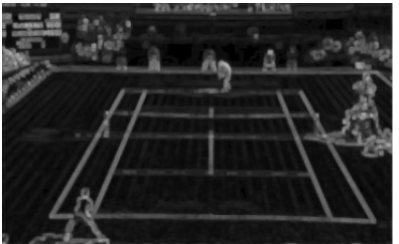

(d)

Figure 3. (a) Original image frame

(b) Edge map using STFHI (c) Pixel intensified frame (d) Edge dilated frame

\subsection{Computing Edge Map Similarity}

The similarity between two edge maps can be considered as computing the similarity of two 2D matrices. Therefore, computing the edge map similarity can be achieved by finding the correlation between the matrices of the edge maps. Computing the Pearson's correlation coefficient $\left(\rho_{X, Y}\right)$ between two matrices $X$ and $Y$ of same dimensions, may be represented as: 
$\rho_{X, Y}=\frac{\sum\left(x_{i j}-\bar{x}\right)\left(y_{i j}-\bar{y}\right)}{\sqrt{\left\{\sum\left(x_{i j}-\bar{x}\right)^{2}\right\}\left(\sum\left(y_{i j}-\bar{y}\right)^{2}\right\}}}=\frac{\sum x_{i j} y_{i j}-\frac{\sum x_{i j} \sum y_{i j}}{n}}{\sqrt{\left\{x_{i j}{ }^{2}-\frac{\left(\sum x_{i j}{ }^{2}\right)}{n}\right\}\left\{y_{i j}{ }^{2}-\frac{\left(\sum y_{i j}{ }^{2}\right)}{n}\right\}}}$

where $x_{i j}$ and $y_{i j}$ are the elements in the $i^{\text {th }}$ row and $j^{\text {th }}$ column of matrices $X$ and $Y$ respectively, $\bar{x}$ is the mean value of elements of $X, \bar{y}$ is the mean value of elements of $Y$ and $n$ is the total number of elements in the matrix under consideration. The correlation is defined only if both of the standard deviations are finite and both of them are nonzero. The correlation is 1 in the case of an increasing linear relationship, -1 in the case of a decreasing linear relationship, and some value in between in all other cases, indicating the degree of linear dependence between the matrices. It is appropriate to mention here that a high value of correlation value indicates high similarity between the image frames.

\subsection{Three Sigma Rule}

The standard deviation $(\sigma)$ of a dataset or probability distribution denotes the variation or deviation from the arithmetic mean $(M)$ or expected value. The three-sigma rule in statistics is used to signify the range in which the values of a normal distribution will lie. According to this rule (refer figure 4), 68.2\% values in a normal distribution lie in the $\operatorname{range}_{[M-\sigma, M+\sigma]}, 95.4 \%$ values in $[M-2 \sigma, M+2 \sigma]$ and $99.6 \%$ in the range $[M-3 \sigma, M+3 \sigma]$. Hence, this empirical rule may be reliably used to compute a threshold to detect values which represent abrupt changes. In the proposed method, three-sigma rule has been used to detect the hard cuts at shot boundaries.

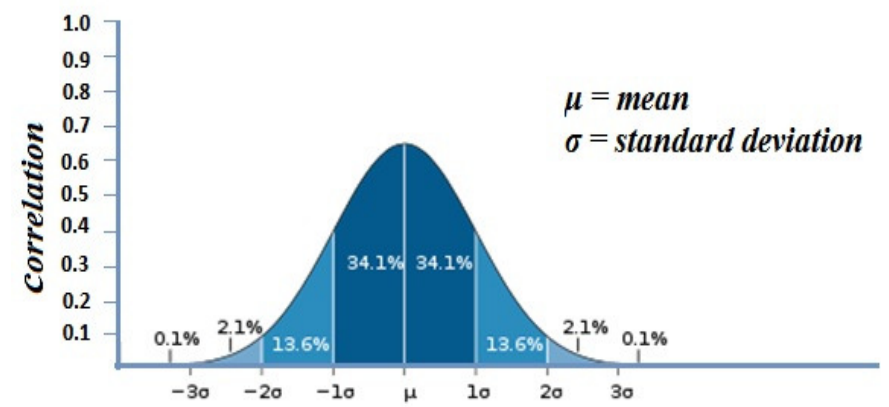

Figure 4. Normal Distribution with three standard deviations from mean

\subsection{Real time updating of parameters used for dynamic threshold}

The correlation between the edge maps of consecutive image frames provides an indication about the similarity of the video frames. However, for detection of a video segment, the correlation gradient is computed from the stream of consecutive correlation values obtained. As mentioned in the previous sub-section, the threshold is computed from the correlation gradient values using the three sigma rule. The parameters involved in calculating the threshold are mean $(M)$ and standard deviation $(\sigma)$ of the correlation gradient values. It must be noted that these parameters are to be updated in real time as new video frames are streamed in. The new mean $\left(M_{n e w}\right)$ and $\left(\sigma_{\text {new }}\right)$ standard deviation may be obtained as follows:- 
$M=\frac{\sum_{i=1}^{N} C_{i}}{N}$ where, $C_{i}$ is the correlation gradient and $N$ is the number of correlation gradient values calculated from the frames received. On arrival of a new frame the value of the new mean will be:-

$M_{\text {new }}=\frac{\sum_{i=1}^{N+1} C_{i}}{N+1}=\frac{\sum_{i=1}^{N+1} C_{i}+C_{N+1}}{N+1}=\frac{M N+C_{N+1}}{N+1} \Rightarrow M_{\text {new }}=\left(\frac{N}{N+1}\right) M+\left(\frac{1}{N+1}\right) C_{N+1}$

The new value of standard deviation $\left(\sigma_{\text {new }}\right)$ may be calculated as follows:-

$$
\begin{aligned}
& \text { Since, } \sigma=\sqrt{\frac{\sum\left(C_{i}-M\right)^{2}}{N}} \Rightarrow N \sigma^{2}=\sum\left(C_{i}-M\right)^{2} \\
& \Rightarrow(N+1) \sigma_{\text {new }}^{2}=\sum_{i=1}^{N+1}\left(C_{i}-M_{\text {new }}\right)^{2} \Rightarrow(N+1) \sigma_{\text {new }}^{2}=\sum_{i=1}^{N+1} C_{i}^{2}-(N+1) M_{\text {new }}^{2} \\
& \Rightarrow \sigma_{\text {new }}^{2}=\frac{1}{N+1}\left[\sum_{i=1}^{N} C_{i}^{2}+C_{N+1}^{2}\right]-M_{\text {new }}^{2}
\end{aligned}
$$

Thus, equations (4) and (5) may be used to update the parameters required for calculating the new threshold. The two equations are significant because the new values of mean and standard deviation can be calculated in real time from the new correlation value computed and earlier values of the two parameters, without having to recalculate the parameters over the whole span.

\section{PROPOSED METHOD FOR REAL TIME VIDEO SEgMENTATION}

The proposed detection mechanism for real time video shot segmentation is shown as a flow diagram in figure 5 . The steps are described in the following subsections.

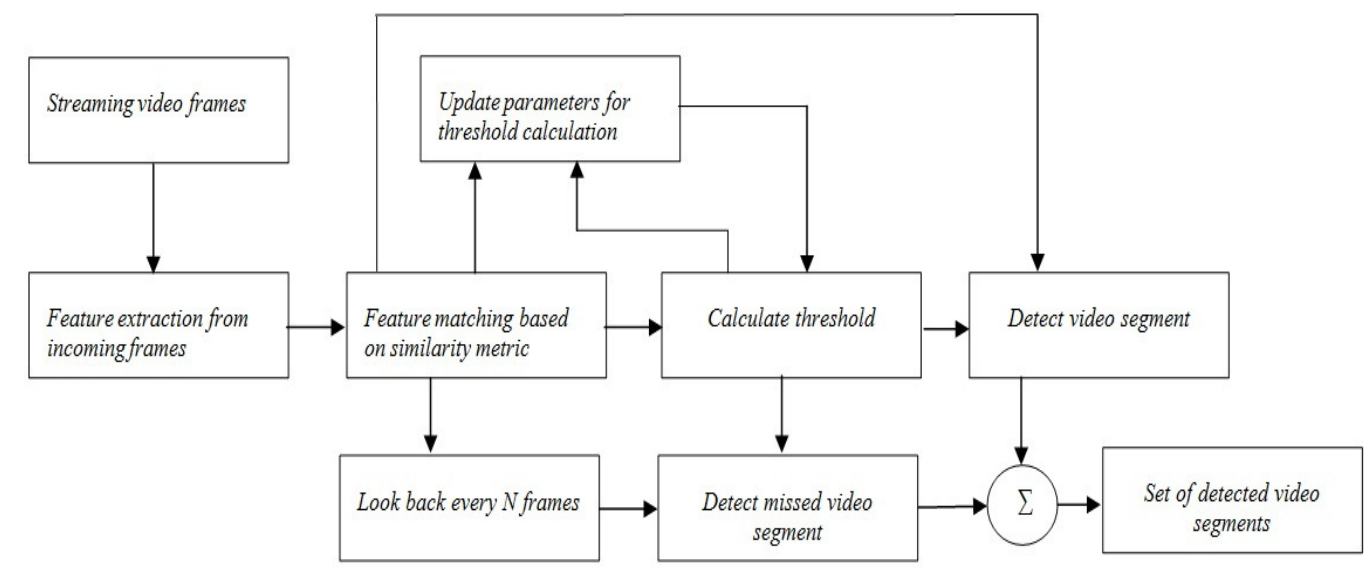

Figure 5. Flow diagram of the video segment detection process in real time

\subsection{Extraction of time sequenced image frames from a video}

The streamed video frames are decomposed into its constituent image frames in a time sequenced manner by a standard codec corresponding to the file type i.e. AVI, MPEG, MP4 etc. The extracted images are in uncompressed format and are stored as bitmaps for further processing. 


\subsection{Feature extraction from the image frames}

The feature extraction process consists of generating a fuzzy hostility map using the STFHI for each image frame, as explained in section 2.2. The fuzzy hostility map indicates the amount of coherence/incoherence in the movement of the objects and is a $2 \mathrm{D}$ matrix which is used to generate the edges of the objects of the gray scale image. Thereafter, an intensity scaling function is used to make the edges more prominent as explained in section 2.3. To compensate for the rapid object or camera movement, edge dilation is performed as explained in section 2.4.

\subsection{Feature matching based on similarity metric}

In this step, the Pearson's correlation between successive fuzzy hostility maps is used as the similarity metric as explained in section 2.5 . The correlation values thus computed are stored in a row matrix $C_{M}$. The shot boundaries occur at points of abrupt change in the correlation values. In order to detect a shot boundary, the gradient of the correlation values (which is a row vector) is computed. The correlation gradient plot is depicted in figure 6, consists of steep spikes at the points of shot boundary.

\subsection{Calculation of threshold and updating of threshold parameters}

Segments in the streaming video are detected by using the three-sigma rule as explained in section 2.6. If the correlation gradient exceeds the upper or lower threshold, a video segment is detected. Since threshold is a function of mean and standard deviation, it has to be updated as new frames are streamed in real time. The new threshold is calculated using equations (4) and (5) explained in section 2.7 .

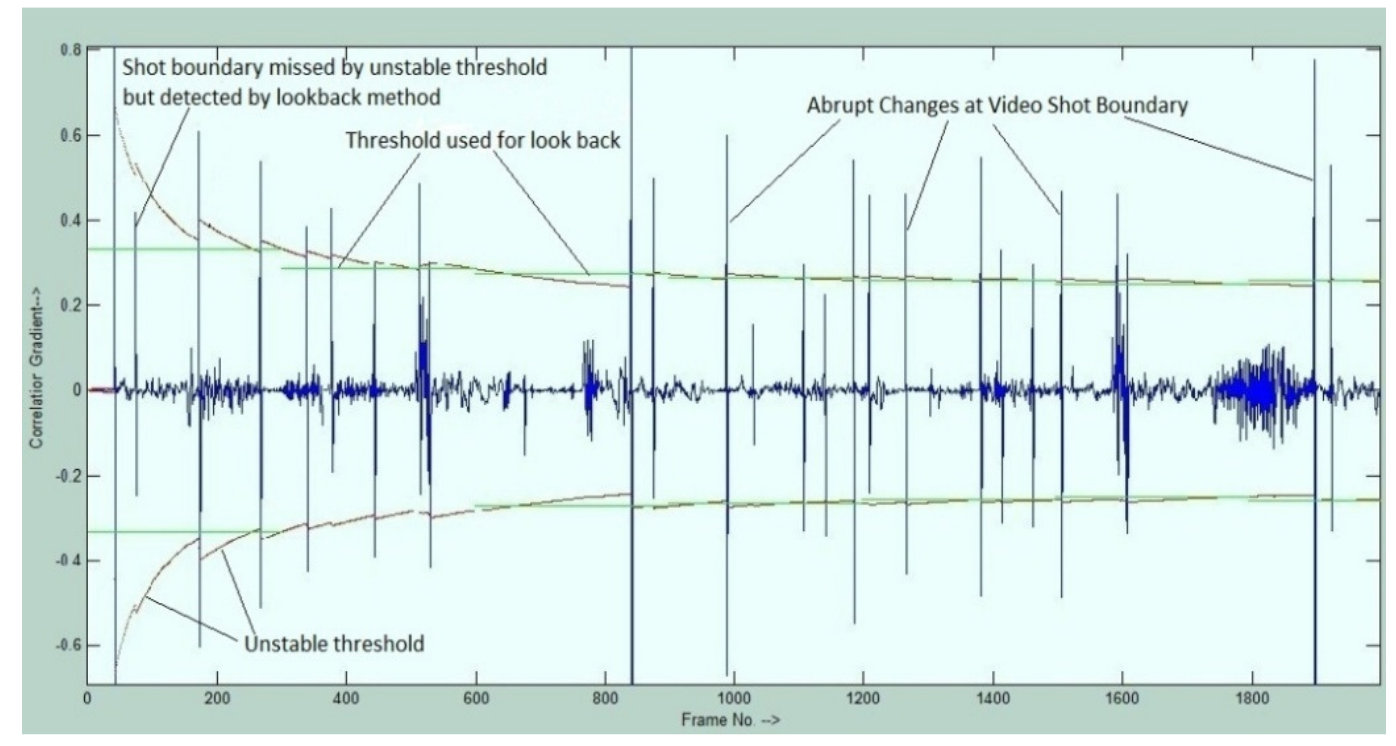

Figure 6. Plot of Correlation gradient values.

\subsection{Look back $\mathbf{N}$ frames to detect missed shot boundaries}

In the proposed video shot detection mechanism, the threshold for detection of shot boundary is set dynamically without any prior knowledge about the type or content of the video. At the initial stage, the threshold fluctuates due to smaller number of data points owing to the lesser number of 
arrived video frames as shown in figure 6 . However, the threshold becomes more stable as more frames arrive in real time. Although the threshold is updated with the arrival of each frame, but the system looks back only after the passage of $N$ frames, to check if any shot boundaries have been missed due to the unstable threshold. In this work, $N=300$ has been taken, i.e. look back will occur every 12 seconds in a video with frame rate 25 fps or every 10 seconds for 30 fps video.

\section{EXPERIMENTAL RESULTS AND ANALYSIS}

The proposed method for shot segmentation in real time videos has been tested on a video data set consisting of eleven videos with varied features (Table I and Table II). All the videos in the test set have a resolution of $640 \times 360$ at $25 \mathrm{fps}$ and are in MP4 format. The performance of the proposed method is evaluated by taking into consideration two parameters, recall $(R)$ and precision $(P)$ defined as follows:-

Recall $=\left(B_{d}-B_{f}\right) / B_{t}$

Precision $=\left(B_{d}-B_{f}\right) / B_{d}$

where, $B_{d}$ : Shot boundaries detected by algorithm; $B_{f}$ : False shot boundaries detected and $B_{t}$ : Actual shot boundaries present in the video

\subsection{The Video Dataset}

The proposed method has been tested on a dataset consisting of two subsets. The first subset comprised five videos which were of long duration of average length of more than one hour (Table 1). The four videos V1 to V4 are documentaries taken from different TV channels. The video V5 is a highlights video taken from the Cricket World Cup 2011 final match between India and Sri Lanka.

The second subset composed of short videos (Table 2). The videos V6 and V7 are sports videos from cricket and tennis. The reason for including this in the dataset was because of the rapid object movement and small length shots. In contrast, V8 and V9 are movie songs from Hindi films. V8 has shots taken mostly outdoors in daylight whereas V9 has real life shots are mixed with some computer generated frames. The average shot duration in V9 is the least among all videos of the dataset. The video V10 is based on a violin track by Lindsey Stirling which is characterized by simultaneous movement of the performer as well as camera. The motivation for including this video is the rapid zoom-in and zoom-out sequences. The video V11 is the official song of the 2010 FIFA World Cup called "Waka Waka". The video comprises of varied background and illumination, intermixed with match sequences taken from FIFA World Cup history.

Table 1. Test Video Dataset-I

\begin{tabular}{|l|l|l|l|l|l|}
\hline Video & V1 & V2 & V3 & V4 & V5 \\
\hline Duration (mm:ss) & $51: 20$ & $28: 40$ & $58: 06$ & $59: 29$ & $111: 19$ \\
\hline No. of Frames & 74020 & 43018 & 87150 & 89225 & 166990 \\
\hline No. of Hard Cuts & 941 & 406 & 807 & 1271 & 2807 \\
\hline $\begin{array}{l}\text { Average no. of frames } \\
\text { in each shot }\end{array}$ & 78.57 & 105.69 & 107.85 & 70.14 & 59.46 \\
\hline
\end{tabular}


Table 2. Test Video Dataset-II

\begin{tabular}{|l|l|l|l|l|l|l|}
\hline Video & V6 & V7 & V8 & V9 & V10 & V11 \\
\hline Duration (mm:ss) & $02: 33$ & $02: 58$ & $02: 42$ & $04: 10$ & $03: 27$ & $03: 31$ \\
\hline No. of Frames & 3833 & 4468 & 4057 & 6265 & 4965 & 5053 \\
\hline No. of Hard Cuts & 46 & 43 & 70 & 172 & 77 & 138 \\
\hline $\begin{array}{l}\text { Average no. of } \\
\text { frames in each shot }\end{array}$ & 81.55 & 101.54 & 57.14 & 36.21 & 63.65 & 36.35 \\
\hline
\end{tabular}

\subsection{Experimental Results}

The proposed method for video shot segmentation is found to work accurately on video frames streamed in at real time. The results obtained by performing the experiments on the video data set are summarized in Table 3. The shot boundaries obtained are the summation of two phases. Video segments are detected using the threshold and method as explained in sections 3.2, 3.3 and 3.4. However some shot boundaries may be missed due to unstable mean and standard deviation. These missed shot segments are detected by updating the threshold and looking back after every $N$ frames have elapsed. The methodology has been discussed in section 3.5. Effectiveness of the proposed method is seen from the high recall and precision values obtained for each of the videos in the test set.

Table 3. Experimental Results of Test Video Dataset

\begin{tabular}{|l|l|l|l|l|l|l|l|l|l|l|l|}
\hline Video & V1 & V2 & V3 & V4 & V5 & V6 & V7 & V8 & V9 & V10 & V11 \\
\hline $\begin{array}{l}\text { Hard Cuts } \\
\text { present }\end{array}$ & 941 & 406 & 807 & 1271 & 2807 & 46 & 43 & 70 & 172 & 77 & 138 \\
\hline $\begin{array}{l}\text { Hard Cuts } \\
\text { detected }\end{array}$ & 940 & 406 & 806 & 1269 & 2796 & 45 & 43 & 67 & 165 & 75 & 136 \\
\hline $\begin{array}{l}\text { Detected by } \\
\text { Lookback }\end{array}$ & 1 & 0 & 1 & 2 & 5 & 2 & 0 & 2 & 5 & 1 & 3 \\
\hline False detection & 0 & 0 & 0 & 0 & 1 & 2 & 0 & 1 & 1 & 0 & 1 \\
\hline Recall (\%) & 100 & 100 & 100 & 100 & 99.75 & 97.82 & 100 & 97.14 & 98.25 & 98.70 & 100 \\
\hline Precision (\%) & 100 & 100 & 100 & 100 & 99.96 & 95.74 & 100 & 98.55 & 99.41 & 100 & 99.28 \\
\hline
\end{tabular}

\subsection{Comparison with other existing methods}

Several existing methods for video segmentation like Mutual Information (MI) [8], Edge Change Ratio (ECR) [13] and Color Histogram Differences (CHD) [14] have been applied for non-real time videos. Shot boundary detection for streaming videos is hard to find in the literature. The problem of automatic computation of a threshold has been addressed in the literature $[1,15]$ and strength of the proposed method lies in the fact that the threshold is computed and updated automatically without manual intervention, unlike the other existing methods. Hence, this method can be applied for on-the-fly detection of shot boundaries in real time videos. The comparative results of the proposed method with its non-real time counterparts are shown in Table 4. 
Table 4. Comparison with Existing Methods

\begin{tabular}{|c|c|c|c|c|c|c|c|c|}
\hline \multirow{2}{*}{ Video } & Proposed Method & \multicolumn{2}{c|}{ MI } & \multicolumn{2}{c|}{ CHD } & \multicolumn{2}{c|}{ ECR } \\
\cline { 2 - 9 } & R & P & R & P & R & P & R & P \\
\hline V1 & $100 \%$ & $100 \%$ & $85.97 \%$ & $92.98 \%$ & $75.98 \%$ & $95.96 \%$ & $90.96 \%$ & $92.98 \%$ \\
\hline V2 & $100 \%$ & $100 \%$ & $83 \%$ & $88.91 \%$ & $78.07 \%$ & $87.93 \%$ & $92.11 \%$ & $96.05 \%$ \\
\hline V3 & $100 \%$ & $100 \%$ & $91.94 \%$ & $93.55 \%$ & $83.02 \%$ & $88.97 \%$ & $89.96 \%$ & $85.99 \%$ \\
\hline V4 & $100 \%$ & $100 \%$ & $87.96 \%$ & $95.98 \%$ & $76 \%$ & $91.03 \%$ & $95.98 \%$ & $92.99 \%$ \\
\hline V5 & $99.75 \%$ & $99.96 \%$ & $85.99 \%$ & $93.97 \%$ & $81.97 \%$ & $91.98 \%$ & $87.99 \%$ & $94.01 \%$ \\
\hline V6 & $97.82 \%$ & $95.74 \%$ & $86.95 \%$ & $90.90 \%$ & $73.91 \%$ & $80.95 \%$ & $91.30 \%$ & $95.45 \%$ \\
\hline V7 & $100 \%$ & $100 \%$ & $88.37 \%$ & $92.68 \%$ & $81.57 \%$ & $96.87 \%$ & $90.69 \%$ & $88.63 \%$ \\
\hline V8 & $97.14 \%$ & $98.55 \%$ & $91.42 \%$ & $94.11 \%$ & $75.71 \%$ & $92.98 \%$ & $95.71 \%$ & $89.33 \%$ \\
\hline V9 & $98.25 \%$ & $99.41 \%$ & $84.88 \%$ & $94.80 \%$ & $77.90 \%$ & $94.36 \%$ & $92.44 \%$ & $88.33 \%$ \\
\hline V10 & $98.70 \%$ & $100 \%$ & $81.81 \%$ & $92.64 \%$ & $74.02 \%$ & $95 \%$ & $93.50 \%$ & $90 \%$ \\
\hline V11 & $100 \%$ & $99.28 \%$ & $84.05 \%$ & $95.08 \%$ & $80.43 \%$ & $94.87 \%$ & $94.92 \%$ & $91.60 \%$ \\
\hline
\end{tabular}

\section{CONCLUSIONS AND REMARKS}

The proposed method for real time video segmentation was tested on a diverse video test set. It is seen to outperform the existing methods in terms of both the recall and precision. As compared to the state-of-the-art techniques, the proposed method achieves nearly 100\% accuracy in terms of both recall and precision. Also, the number of false detections is very less. The problem of automatically setting the threshold, without any human interference, has been addressed and results are very encouraging. The number of false hits is almost negligible as compared to the other existing methods. A major challenge would be to detect dissolve edits in streaming videos.

\section{ACKNOWLEDGEMENTS}

The authors acknowledge the contributions of Surajit Dutta, M.Tech student at RCC Institute of Information Technology, Kolkata, during the experimentation phase of the proposed method.

\section{REFERENCES}

[1] Alan Hanjalic "Shot-Boundary Detection: Unraveled and Resolved," Circuits and Systems for Video Technology, IEEE Transactions, Volume:12, Page(s):90-105, February 2002.

[2] Siddhartha Bhattacharyya, Ujjwal Maulik, and Paramartha Dutta "High-speed target tracking by fuzzy hostility-induced segmentation of optical flow field," Applied Soft Computing ,Science Direct, 2009.

[3] Hattarge A.M., Bandgar P.A., and Patil V.M. "A Survey on Shot Boundary Detection Algorithms and Techniques”, International Journal of Emerging Technology and Advanced Engineering, Volume 3, Issue 2, February 2013.

[4] Biswanath Chakraborty, Siddhartha Bhattacharyya, and Susanta Chakraborty "A Comparative Study of Unsupervised Video Shot Boundary Detection Techniques Using Probabilistic Fuzzy Entropy Measures”, DOI: 10.4018/978-1-4666-2518-1.ch009 in Handbook of Research on Computational Intelligence for Engineering, Science, and Business, 2013.

[5] John S. Boreczky, and Lawrence A. Rowe "Comparison of video shot boundary detection techniques", Journal of Electronic Imaging, Page(s):122-128, March,1996.

[6] Swati D. Bendale, and Bijal. J. Talati "Analysis of Popular Video Shot Boundary Detection Techniques in Uncompressed Domain,” International Journal of Computer Applications (0975 8887), Volume 60- No.3, IJCA, December, 2012. 
[7] Ullas Gargi, Rangachar Kasturi, and Susan H. Strayer "Performance Characterization of Video-ShotChange Detection Methods," IEEE Transaction on Circuits and Systems for Video Technology, Vol. 10, No. 1, IEEE, February 2000.

[8] Aarti Kumthekar and Mrs.J.K.Patil "Comparative Analysis Of Video Summarization Methods," International Journal of Engineering Sciences \& Research Technology, ISSN: 2277-9655,2(1): Page(s): 15-18, IJESRT January, 2013.

[9] R. Lienhart, S. Pfeiffer, and W. Effelsberg "Scene determination based on video and audio features," Proceedings of IEEE International Conference on Multimedia Computing and Systems, Volume:1, Page(s):685 -690 IEEE, 1999.

[10] H. Zhang, A. Kankanhalli, and S.W. Smoliar "Automatic partitioning of full-motion video," Multimedia Systems, Volume: 1, no. 1, Page(s): 10-28, 1993.

[11] A. Nagasaka and Y. Tanaka "Automatic video indexing and full-video search for object appearances," Proceedings of IFIP 2nd Working Conference on Visual Database Systems, Page(s): 113-127, 1992

[12] A. Hampapur, R. C. Jain, and T. Weymouth "Production Model Based Digital Video Segmentation," Multimedia Tools and Applications, Vol.1, No. 1, Page(s): 9-46, March 1995.

[13] R. Zabih, J. Miller, and K. Mai "A Feature-Based Algorithm for Detecting and Classifying Scene Breaks," Proceedings of ACM Multimedia 1995, San Francisco, CA, Page(s):189-200, November, 1995.

[14] Vrutant Hem Thakore "Video Shot Cut Boundary Detection using Histogram," International Journal of Engineering Sciences \& Research Technology, ISSN: 2277-9655, 2(4): Page(s): 872-875, IJESRT, April, 2013.

[15] L. Ranathunga, R. Zainuddin, and N. A. Abdullah "Conventional Video Shot Segmentation to Semantic Shot Segmentation," 6th IEEE International Conference on Industrial and Information Systems (ICIIS), Page(s):186-191, August 2011.

\section{AUTHORS}

Hrishikesh Bhaumik is currently serving as an Associate Professor and HOD of Information Technology Department at RCC Institute of Information Technology, Kolkata, India. He did B.Sc. from Calcutta University in 1997, AMIE in Electronics and Comm. Engg in 2000 and M.Tech in Information Technology from Bengal Engineering and Science University, Shibpur in 2004. In 2008 he received sponsorship for working on a Business Process Sniffer Tool developed at Infosys, Bhubaneswar. He made significant contributions to the EU-INDIA grid project in 2010 and 2011. His research interests include Content Based Video Retrieval Systems, Text Mining and High Performance Computing.

Siddhartha Bhattacharyya did his Bachelors in Physics, Bachelors in Optics and Optoelectronics and Masters in Optics and Optoelectronics (Gold Medal) from University of Calcutta, India in 1995, 1998 and 2000 respectively. He completed PhD (Engg.) in Computer Science and Engineering from Jadavpur University, India in 2008. He is currently an Associate Professor and Dean (R \& D) in the Department of Information Technology of RCC Institute of Information Technology, Kolkata, India. He is a co-author of two books, co-editor of a book and has more than 90 research publications. He was the

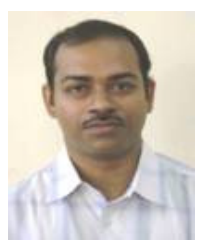
member of the Young Researchers' Committee of the WSC 2008 Online World Conference on Soft Computing in Industrial Applications. He was the convener of the AICTE-IEEE National Conference on Computing and Communication Systems (CoCoSys-09) in 2009. He is the Assistant Editor of International Journal of Pattern Recognition Research since 2010. He is the Associate Editor of International Journal of BioInfo Soft Computing since 2013. He is the member of the editorial board of International Journal of Engineering, Science and Technology and the member of the editorial advisory board of HETC Journal of Computer Engineering and Applications. He is a senior member of IEEE and member of ACM, IRSS and IAENG. He is a life member of OSI and ISTE, India.His research interests include soft computing, pattern recognition and quantum computing. 
Dr. Susanta Chakraborty received the B. Tech, M.Tech and Ph.D(Tech) in Computer Science in 1983, 1985 and 1999 respectively from the University of Calcutta. He is currently a Professor in the department of Computer Science and Technology at the Bengal Engineering Science and University, Shibpur, West Bengal, India. Prior to this he served at University of Kalyani as a Dean of Engineering, Technology and Management faculty. He has published around 31 research papers in reputed International Journals including IEEE Transactions on CAD and refereed international conference proceedings of IEEE

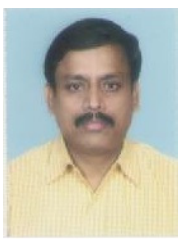
Computer Science Press. He was awarded INSA-JSPS Fellowship of Indian National Science Academy (INSA) in the session 2003-2004. He has collaborated with leading scientists around the world in areas of Test Generation of Sequential Circuits and Low Power Design, VLSI testing and fault diagnosis, Quantum circuit and Testable Design of Nano-Circuits and Micro Fluidic Bio-chips. He has more than 25 years of research experience. He has served as the Publicity Chair, Publication Chair and Program Committee members of several International Conferences. 